Rev Biomed 2001; 12:186-195.

\title{
Fundamentos y actualidades del asesoramiento genético.
}

\section{Revisión}

Doris Pinto-Escalante, José M. Ceballos-Quintal, Ileana Castillo-Zapata, María T. de J. López-Avila.

Laboratorio de Genética, Centro de Investigaciones Regionales "Dr Hideyo Noguchi”, Universidad Autónoma de Yucatán, Mérida, Yucatán, México.

\section{RESUMEN.}

Los avances en el conocimiento de los mecanismos de producción de las enfermedades hereditarias, y la aplicación de los principios de genética en diferentes disciplinas no tratadas de manera común por los genetistas, han ampliado la utilidad del asesoramiento genético. Por estas razones, en la actualidad es mayor el número de familias que buscan asesoría genética por defectos al nacimiento, enfermedades genéticas, así como por causas tan diversas como cáncer, trastornos psiquiátricos, neurodegenerativos, cardiacos, abortos, óbitos, embarazo en edad avanzada, exposición a teratógenos y otros factores de riesgo.

$\mathrm{El}$ asesoramiento genético es un proceso de comunicación acerca de los problemas humanos asociados con la ocurrencia, o riesgo de ocurrencia y repetición, de un desorden genético familiar. Debe ser proporcionado con imparcialidad, para alcanzar la finalidad de ayudar al individuo, o la familia, para afrontar su situación relacionada con la enfermedad, por medio de información clara para comprender los aspectos médicos, modo de herencia y riesgos de repetición que les permita tomar decisiones razonadas acerca del motivo de su consulta.

En esta revisión se abordan los fundamentos en los que se basa el asesoramiento genético, las características de los diferentes modos de herencia tradicionales (herencia mendeliana, cromosómica y multifactorial), novedosos (inestabilidad del genoma, herencia mitocondrial, cambios epigenéticos), y teratogénicos, los principios éticos elementales relacionados con este tema, y se hacen consideraciones acerca de la necesidad de formación de profesionales en salud entrenados para proporcionar consejo genético bajo la supervisión de genetistas clínicos.

(Rev Biomed 2001; 12:186-195)

Palabras clave: Asesoramiento genético, consejo genético, riesgo genético, ética.

Solicitud de sobretiros: Dra. Doris Pinto-Escalante, Laboratorio de Genética, Centro de Investigaciones Regionales “Dr Hideyo Noguchi”, Universidad Autónoma de Yucatán. Calle 59 No. 490 x Av. Itzáes, C.P. 97000, Mérida, Yucatán, México. e-mail: pescalan@tunku.uady.mx Recibido el 25/Febrero/2000. Aceptado para publicación el 9/Mayo/2000. 


\section{Pinto-Escalante, JM Ceballos-Quintal, I Castillo-Zapata, MT de J López-Avila.}

\section{SUMMARY.}

\section{Foundation and actuality about genetic counseling.}

Advances in the knowledge of mechanisms responsible for hereditary diseases, and the application of genetic principles in areas not common for geneticists, has widened the use of genetic counseling. For these reasons, there is now a higher number of families who look for genetic counseling because of congenital malformations, genetic disorders, and other causes such as cancer, psychiatric, neurodegenerative and cardiac disorders, multiple miscarriages, stillbirths, advanced age pregnancy, teratogen exposition and other risk factors.

Genetic counseling is a communication process which deals with human problems associated with the occurrence, or the risk of occurrence and recurrence, of a genetic disorder in a family. It must be provided with impartiality in order to help the individual, or the family, to confront their situation in relation to the disease, by providing information to help them comprehend medical facts, heredity contribution to the disorder, and recurrence risks, so they can make wellinformed reasoned decisions.

In this work we describe the principles which support genetic counseling, the characteristics of traditional genetic diseases (mendelian, chromosomic and multifactorial inheritance), new concepts (instability of the genome, mitochondrial inheritance, epigenetic changes), and teratogenic substances. Ethical principles in relation to this topic are discussed, and we make considerations about the need for the postgraduate education of health professionals trained to provide genetic counseling under the supervision of clinical geneticists. (Rev Biomed 2001; 12:186-195)

Key words: genetic counseling, genetic risk, ethic.

\section{INTRODUCCIÓN.}

$\mathrm{El}$ asesoramiento genético (AG), o consejo genético, trata de la información referente a la etiología, historia natural y riesgo de repetición de enfermedades hereditarias (1). Es un proceso de comunicación acerca de los problemas humanos asociados con la ocurrencia, o riesgo de ocurrencia, de un desorden genético familiar. La finalidad de la información es ayudar al individuo, $\mathrm{o}$ a la familia, para afrontar su situación relacionada con la enfermedad por la que consulta. Debido a la complejidad que puede tener su problemática, el asesoramiento genético debe incluir suficiente información, de tal manera que quien la recibe pueda $(2,3)$.

Comprender los aspectos médicos, incluyendo el diagnóstico, curso probable de la enfermedad y manejo posible.

Apreciar la forma en que la herencia contribuye para producir la enfermedad y en el riesgo de repetición.

Entender las posibilidades del riesgo de repetición.

Escoger el curso de acción que parece apropiado debido al riesgo, metas familiares, estándares éticos y religiosos y actuar de acuerdo con esta decisión.

Hacer el mejor ajuste posible a la patología en un miembro afectado de la familia y/o al riesgo de repetición de este desorden.

El AG se desarrolló en el contexto del cuidado materno y pediátrico, por la participación de los defectos al nacimiento y las hospitalizaciones pediátricas debidas a alteraciones genéticas como causa principal o componente parcial (4). Los defectos al nacimiento y las enfermedades genéticas formaron los grupos principales a quienes estaba dirigido el AG, sin embargo, actualmente es mayor el número de familias que buscan asesoramiento por la posibilidad de riesgo a diferentes características hereditarias normales o anormales, ciertos cánceres, trastornos psiquiátricos, neurodegenerativos y cardíacos. También quedan incluidas las parejas con historia de múltiples abortos, nacidos muertos o muertes tempranas, mujeres que pretenden embarazarse o que están

\section{Revista Biomédica}


embarazadas en edad avanzada, o por exposición gestacional a posibles teratógenos. Así mismo, personas de ciertos grupos étnicos o áreas geográficas con mayor incidencia a patologías específicas forman un grupo adicional que actualmente busca orientación por medio del AG.

Tradicionalmente el AG se proporciona, en general en todo el mundo, por médicos genetistas o médicos con conocimientos avanzados en genética. Sin embargo, debido a la diversificación de los motivos por los que se busca asesoría genética, y por los avances recientes en el diagnóstico de patologías con componente genético parcial, este proceso de información se ha extendido a diferentes disciplinas que no son de manejo predominante del genetista. Los avances en el conocimiento de la genética han creado la necesidad de proporcionar AG en todas las áreas del cuidado primario de la salud y en especialidades diversas, como la oncología y cardiología, por lo que se ha abierto la posibilidad de que el $\mathrm{AG}$, al menos en estos campos u otras similares, sea proporcionado en el futuro por médicos u otro personal de salud preparado específicamente para este fin. Así mismo, el diagnóstico prenatal de múltiples patologías en el producto en desarrollo, también ha contribuido a que el AG se diversifique a este campo en el que adquiere características particulares.

El proyecto Genoma Humano ha contribuido de manera particular a que la genética clásica, basada principalmente en la herencia Mendeliana y aberraciones cromosómicas, amplíe su campo de conocimientos e incluya formas de herencia notradicionales, como la inestabilidad del genoma, herencia no nuclear, cambios epigenéticos en forma de imprinting y herencia conformacional de proteínas. Las formas no tradicionales de herencia resultan cada vez mas importantes para la patología humana. Estos conceptos requieren ser difundidos por su utilización cada vez más frecuente en múltiples áreas de la medicina, y por sus implicaciones en el AG.

Esta revisión tiene como finalidad describir de una manera sencilla esta terminología, ejemplificarla con patologías relacionadas y las características generales de asesoramiento para cada caso. Iniciamos con los conceptos de la herencia clásica hasta concluir con las implicaciones que conlleva el AG en el aspecto ético.

\section{Herencia Mendeliana}

En este grupo quedan incluidas las enfermedades ocasionadas por mutaciones en un solo gen, que puede estar localizado en autosomas o en cromosomas sexuales. La característica del comportamiento del gen, como dominante o recesivo, así como la presencia de la mutación en un solo alelo o en ambos de cada par de cromosomas, permiten identificar características distintivas para cada una de las cuatro variedades de herencia mendeliana: autosómica dominante y recesiva, y ligadas al $\mathrm{X}$ dominante y recesiva, cada una con árboles genealógicos con particularidades que permiten por sí mismos indicar el tipo de herencia. Además, se pueden establecer los riesgos de repetición con base en el modo de herencia identificado.

En el AG de las cuatro variedades de herencia mendeliana, cada vez que ocurre un embarazo debe considerarse como un evento independiente. Es decir, una vez que se sabe que una pareja se encuentra en riesgo de tener descendencia genéticamente afectada, este riesgo se presenta con cada embarazo. El hecho de haber tenido un hijo previo afectado, no lo excluye de que el siguiente pueda estarlo también, como tampoco aumenta o disminuye este riesgo en futuros embarazos. El riesgo de repetición para enfermedades mendelianas se considera alto.

En algunas ocasiones se puede encontrar familias en las que una enfermedad con un modo de herencia reconocido con una variedad de herencia mendeliana particular, tiene un comportamiento de repetición atípico. Por ejemplo, una enfermedad autosómica dominante en un afectado con ambos progenitores sanos, debe considerarse que ocurrió por una mutación de novo

Vol. 12/No. 3/Julio-Septiembre, 2001 


\section{Pinto-Escalante, JM Ceballos-Quintal, I Castillo-Zapata, MT de J López-Avila.}

y por tanto los padres no tendrían riesgo de tener otro hijo afectado. Sin embargo, cuando en familias como ésta se presenta nuevamente la enfermedad en otros hijos, debe considerarse la posibilidad de un mosaicismo (dos líneas celulares, una normal y otra con la mutación que produce el gen anormal) parental que involucre la línea celular germinal (gónadas). Esta situación ocurre rara vez, en estas familias el riesgo de tener otro hijo afectado es mayor que para la población general, pero menor al del modo de herencia identificado para la patología específica.

En general se puede considerar que las patologías con modo de herencia mendeliano no presentan mayor dificultad para el AG, en cuanto a riesgos de repetición se refiere (5).

\section{Cromosomopatías}

Las cromosomopatías corresponden a los padecimientos originados por alteraciones de los cromosomas visibles al microscopio y pueden ser de tipo numérico o estructural, que involucre alguno de los 22 pares de autosomas y/o el par sexual.

En las aberraciones cromosómicas, existen diferencias sustanciales en su expresión clínica, dependiendo de que el cromosoma afectado sea un autosoma o un cromosoma sexual. Así mismo, las manifestaciones clínicas varían entre las diferentes alteraciones producidas por los autosomas. Se puede generalizar, con muy pocas excepciones, que las aberraciones que afectan a los autosomas tendrán manifestaciones de deficiencia mental, además de malformaciones mayores o menores. En las aberraciones que involucran algún cromosoma sexual lo que ocurre con mas frecuencia es que resulte afectada la reproducción, con variabilidad en la presencia o no de malformaciones congénitas, pero sin deficiencia mental.

No existe un patrón generalizado de riesgo de repetición para las aberraciones cromosómicas. Es importante valorar cada caso particular, con los antecedentes familiares, la aberración identificada y el cromosoma involucrado, para poder determinar el AG apropiado a la familia. Es crucial para este grupo de patologías contar con un laboratorio confiable de diagnóstico citogenético (6).

Resulta más complejo el AG de cromosomopatías cuando éstas se diagnostican en etapa prenatal, pues puede conducir a que la pareja decida terminar el embarazo. Por esto, resulta todavía más importante conocer las implicaciones de las manifestaciones clínicas y el pronóstico de vida, en tiempo y calidad, del paciente afectado con la anomalía detectada. En estos casos existe mayor tendencia a que la imparcialidad del AG no se cumpla (7-9), En nuestro país aún no existe una práctica generalizada del diagnóstico prenatal citogenético por lo que estos casos difícilmente se presentan para AG.

\section{Herencia multifactorial}

En estos casos, la manifestación clínica de la enfermedad (fenotipo), es resultado de la interacción del efecto acumulado de varios pares de genes, aunado a influencias del medio ambiente. El paciente recibe de sus progenitores predisposición para desarrollar alguna patología, que solo es evidente en un ambiente favorable. Para obtener el riesgo de repetición de este grupo de patologías, por tanto, debe considerarse la "carga" genética del individuo afectado. La carga genética podemos estimarla si conocemos el número de parientes afectados, grado de parentesco, gravedad de la malformación y género de cada afectado. Los rasgos o características de este grupo de patologías se distribuyen en la población de una forma normal (curva en forma de campana) y para que se manifieste debe sobrepasarse un "umbral". Los riesgos específicos para las patologías de este grupo, como labio y paladar hendidos, defectos en el cierre del tubo neural y estenosis pilórica, se han estimado empíricamente con el estudio de múltiples familias afectadas, es variable por cada malformación y carga genética de la familia $(3,10)$.

\section{Revista Biomédica}




\section{Teratógenos}

Se conocen como teratógenos a los factores externos que producen malformaciones congénitas o anomalías funcionales en el embrión o feto en desarrollo. Existe una gran variedad de exposiciones que son potencialmente teratogénicas para el humano, entre las que se incluyen algunos medicamentos, el abuso de drogas recreacionales, agentes químicos, físicos e infecciosos, y estado metabólico de la madre. El efecto de un agente teratogénico debe considerarse en el contexto de las circunstancias de la exposición, que incluye la naturaleza del agente, la dosis de exposición, su ruta y la edad gestacional. También debe valorarse posibles exposiciones simultáneas, la susceptibilidad materna y del producto en desarrollo, para saber si un agente puede producir daño en un momento particular

Las circunstancias que pueden motivar una consulta debido a un agente teratogénico quedan en tres grupos: cuando un paciente presenta alguna malformación y en la historia de su embarazo se identifica la posibilidad de un agente teratógeno. Cuando un paciente que en el curso de un tratamiento o de una exposición ocupacional, planea un embarazo. O cuando una mujer embarazada está interesada por un riesgo teratogénico asociado con una exposición previa $o$ actual. En todos los casos, para el asesoramiento de riesgo, debe valorarse cuidadosamente la interpretación de la literatura acerca del efecto teratogénico del agente de exposición, así como las circunstancias particulares, dosis y tiempo de la exposición y considerar si el riesgo para la malformación es mayor que el de la población general no expuesta (11).

\section{Inestabilidad del genoma}

La anticipación, o empeoramiento de algunas enfermedades genéticas en generaciones subsecuentes, o disminución de la edad de aparición de una enfermedad, era un hecho que se explicaba como un problema de falta de indagación y diagnóstico en las generaciones previas.
Actualmente se sabe que es producto de un fenómeno conocido como inestabilidad del genoma, consecuencia de la inestabilidad de trinucleótidos repetidos. En el genoma humano es normal encontrar segmentos que poseen repetición de tripletes de nucleótidos. En ocasiones puede encontrase mayor número de tripletes que en un alelo normal, que existen en un estado de "premutación", pero incapaces de producir enfermedad. Sin embargo, son inestables y pueden amplificarse durante la división celular por meiosis. El mayor número de tripletes, producto de la amplificación en el estado de premutación, se encuentra asociado a enfermedad. Las mutaciones producidas por expansión de trinucleótidos repetidos son capaces de producir enfermedad, su severidad está en relación directa con el número de repeticiones de los tripletes $(12,13)$.

Es reducido el número de enfermedades genéticas en las que se ha identificado la inestabilidad del genoma como su causa. Cuando menos son 11 los locus identificados con expansión de tripletes en los humanos, a 9 de ellos se les ha atribuido la producción de enfermedades. Entre éstas se encuentran el síndrome de $\mathrm{X}$ frágil, las atrofias musculares espinal y bulbar, la ataxia espinocerebelosa tipo I, la enfermedad de Huntington y la distrofia miotónica.

El síndrome de $X$ frágil, la causa hereditaria más frecuente de retraso mental, está ocasionado por repeticiones del triplete CCG. Está localizada en un sitio frágil del cromosoma X (Xq27.3), observado citogenéticamente como una brecha sin teñir en el cromosoma en metafase. En las personas normales, la repetición de este nucleótido es polimórfica, encontrándose de 6 a 52 copias, se expande preferencialmente durante la meiosis materna hasta encontrarse mas de 200 veces en el estado patológico. En este síndrome las manifestaciones clínicas típicas incluyen retraso mental moderado a severo, facies caracterizada por ser estrecha y alargada, con prominencia frontal y orejas grandes, así como macroorquidismo postpuberal (13).

Vol. 12/No. 3/Julio-Septiembre, 2001 


\section{Pinto-Escalante, JM Ceballos-Quintal, I Castillo-Zapata, MT de J López-Avila.}

Para el AG de este grupo particular de enfermedades, además del modo de herencia mendeliana particular, debe considerarse el número de generaciones afectadas, así como la gravedad y la severidad de los individuos afectados.

Otro grupo de enfermedades en el que se ha encontrado expansión de tripletes es el cáncer, en el que la inestabilidad de éstos que conduce a su amplificación ocurre durante la división celular por mitosis.

La inestabilidad del genoma puede manifestarse también por otros mecanismos, como los transposones. En este fenómeno, el gen se interrumpe por un elemento llamado transposon, que codifica para una transposasa activa, que cambia de sitio segmentos de DNA. Este es uno de los mecanismos que produce mutación en el gen del factor VIII de la coagulación, que al estar alterado produce hemofilia.

\section{Herencia mitocondrial}

El reconocimiento de que las mutaciones en el DNA mitocondrial son capaces de producir enfermedades hereditarias es reciente (1988), presenta características peculiares, debido a que las mitocondrias se heredan por rama materna, y cada célula posee múltiples mitocondrias. Al igual que los padecimientos recesivos ligados al $\mathrm{X}$, la transmisión de las enfermedades mitocondriales es por parte de la madre, aunque en este caso la enfermedad la pueden presentar tanto varones como mujeres. Sin embargo, sólo las hijas pueden transmitir nuevamente la enfermedad a sus hijos e hijas, situación que la diferencía con la herencia mendeliana simple ligada al X recesiva. En general, en la historia familiar se observa asimetría en la severidad esperada entre hombres y mujeres, con efectos severos en varones y moderados en mujeres (14).

Las mutaciones que causan enfermedades mitocondriales presentan manifestaciones clínicas muy variables. La presencia de múltiples mitocondrias en cada célula, no necesariamente todas con la mutación, condiciona que la enfermedad pueda tener diferentes manifestaciones, dependiendo de la relación de mitocondrias con mutación o sin ella (heteroplasmía). Cuando las células solo poseen DNAmt mutante se denominan homoplásticas, en ellas el efecto de la enfermedad es mayor. Así mismo, la heterogeneidad en la distribución del DNA mitocondrial mutado entre diferentes tejidos es otra explicación posible a la amplia variedad de fenotipos en pacientes con desórdenes mitocondriales (15).

Las explicaciones anteriores ocasionan una dificultad grande para conocer la situación individual de cada paciente y poder proporcionar una explicación sobre algunas características que pudiese presentar la enfermedad en su descendencia. Debido a que son muy pocos los casos reconocidos de enfermedades mitocondriales, se necesita mayor información para establecer criterios adecuados para AG.

\section{Cambios epigenéticos}

Las diferencias no heredables en la expresión de genes de una enfermedad se conocen como cambios epigenéticos. Las forma mejor conocida es la impronta genómica.

\section{Impronta genómica:}

El principal cambio epigenético es la impronta genómica, proceso por el cual un gen está inactivado o silenciado, con el resultado de que solo uno de los dos genes normales está activo. Esta diferencia de expresión en los genes a nivel molecular se traduce en la clínica como diferencias de expresión fenotípica. El fenómeno de impronta genómica ocurre durante el desarrollo del óvulo y del esperma. En este período crítico el DNA presenta modificaciones que alteran la expresión de genes de acuerdo al progenitor de origen. Una de las modificaciones mejor conocidas es la metilación del DNA.

En el humano se ha encontrado que los cromosomas 11, 15, 7 y 14 tienen regiones en las que ocurre el fenómeno de impronta. Los genes en los que ocurre la impronta parecen estar

\section{Revista Biomédica}


acumulados y organizados en dominios de estas regiones cromosómicas (16). El número de padecimientos en los que se considera que participa la impronta genómica se ha incrementado con el análisis detallado de la genealogía. La información que debe incluirse para análisis es saber si hay diferencias en la edad de aparición de la enfermedad, en la gravedad de las manifestaciones o en el cuadro clínico y si estas diferencias tienen relación con algún progenitor. La identificación de más padecimientos en los que se identifique el fenómeno de la impronta genómica, como un mecanismo adicional a los clásicos para la producción de enfermedad, debe considerarse para proporcionar el AG. Los riesgos habrán que determinarlos por el género del progenitor transmisor de la enfermedad. El alelo anormal o ausente proviene de la madre en la impronta materna (o de sus hermanas o sus hijas), no se observa expresión fenotípica, el gen está "silenciado", pero se activa al pasar la espermatogénesis (padre, hermanos, hijos varones). Lo opuesto se observa en la impronta paterna, es decir, no habrá expresión fenotípica si la modificación se hereda a través del padre.

El fenómeno de la impronta genómica se observa con claridad en los síndromes de PraderWilli y en el de Angelman. Ambas patologías pueden presentarse por deleción de la región intersticial del brazo largo del cromosoma 15, región 11 a 13. El síndrome de Prader-Willi está caracterizado por hipotonía, detención de crecimiento en la infancia, manos y pies pequeños, hipogonadismo, retraso mental variable y obesidad marcada. El síndrome de Angelman se presenta con retraso mental profundo, facies alegre, marcha atáxica con movimiento amplio de brazos, anormalidades en el EEG, hiperactividad, brotes de risa e hipotonía moderada. El síndrome de Angelman se presenta cuando el cromosoma que perdió el segmento de DNA es de origen materno. Por el contrario, si el cromosoma delecionado fue de origen paterno, la expresión fenotípica es el síndrome de Prader-Willi. Ambas patologías también pueden encontrarse en pacientes sin la deleción, pero que tienen inactivo un gen localizado en ese sitio, $\mathrm{o}$ ambos cromosomas del par provienen de un solo progenitor (disomía uniparental) (1618).

Es importante conocer si existen diferencias en expresión de genes dependiendo del origen parental, para poder conocer los riesgos a los que queda expuesta la pareja al tener descendencia. En el tumor de Willms y otros cánceres también se observa el mecanismo de impronta genómica (19).

\section{Imparcialidad en el asesoramiento genético.}

La imparcialidad para el AG está basada en el deseo de apoyar la decisión reproductiva de la pareja, sin pasar por el juicio de demeritar la vida de una persona afectada por una condición genética. Sin embargo, en la práctica los pacientes pueden estar influenciados por la clase de información proporcionada y por la forma en que es dada. Los pacientes pueden sentirse persuadidos por los informadores para seguir una conducta determinada (20). Debe considerarse que las circunstancias (religiosas, sociales, familiares) de una persona o familia afectada por una enfermedad genética, pueden hacer variar la apreciación que se tenga de la enfermedad y sus posibles alternativas para superarla. Por lo tanto, debe corresponder a la persona asesorada la decisión de la conducta a seguir, después de haber recibido la información suficiente para determinar sus propios juicios con libertad.

En México, la mayoría de los genetistas coinciden en que el AG debe ser imparcial, sin embargo, en situaciones de diagnóstico fetal anormal pueden optar por proponer la interrupción del embarazo o no (9-21). Un estudio realizado con genetistas Mexicanos reveló que la minoría cumple la imparcialidad en el AG cuando se trata de anomalías fetales, y que el consejo estuvo influenciado por la severidad de la patología, con mayor tendencia a la neutralidad por los genetistas con mas años de experiencia (9). 


\section{Pinto-Escalante, JM Ceballos-Quintal, I Castillo-Zapata, MT de J López-Avila.}

\section{Implicaciones éticas}

Es de vital importancia tener presentes ciertos preceptos relacionados con el AG. Entre ellos, la confidencialidad de la información proporcionada por el consultante requiere de especial atención. Esta norma adquiere mayor importancia si consideramos que el incremento de las posibilidades para pruebas genéticas y de tamizaje puede llevarnos a identificar personas sanas, presintomáticas o con mayor riesgo a tener enfermedades genéticas. En la actualidad, las pruebas de tamizaje no se enfocan sólo a enfermedades que ocurren en la familia, sino también en grupos raciales o étnicos de uno o ambos padres. La información que se tenga como resultado de estas pruebas puede conducir a consecuencias serias en el estilo de vida futura, empleo y seguros de vida de la persona estudiada. Por estas razones es de suma importancia mantener la confidencialidad de cualquier información recibida de los consultantes, o de los resultados de sus estudios, a menos que se tenga autorización expresa para darla a conocer (22-25).

En relación a la persona que recibe el $\mathrm{AG}$, el consejero debe tener presente las necesidades de quien lo recibe. La relación entre el consejero y el aconsejado debe estar basada en valores de cuidado y respeto a su autonomía, tener presente su individualidad, bienestar y libertad, así como las tradiciones culturales, inclinaciones, circunstancias y sentimientos. La información que se proporciona debe permitir a quien la escucha, ser capaz de tomar decisiones independientes, libres de imposiciones, a través de ofrecer información clara, o ilustrando los hechos necesarios, clarificando las alternativas y anticipando consecuencias. El impacto psicológico de saber un riesgo genético, y las difíciles decisiones a las que se enfrenta un individuo identificado con riesgo genético, pueden requerir un apoyo mayor al de una sesión de información, con el fin de proporcionar un apoyo que permita superar el impacto de la información (26). Debe tenerse en cuenta que la interpretación subjetiva del paciente, acerca de los riesgos proporcionados en el AG, pueden ser un factor importante que haga una diferencia sustancial en el impacto de ésta.

El análisis de las respuestas de un grupo de genetistas Mexicanos sobre tópicos controversiales, basado en los principios generales de la ética médica: respeto a la persona, beneficencia, no maleficiencia, proporcionalidad y justicia, indican que el asesoramiento genético se proporciona sin imparcialidad, con poca consideración a la autonomía del paciente. Estas actitudes fueron motivadas por el deseo de ayudar a los pacientes al darles el "mejor" consejo, basado en los valores del médico y su percepción de la severidad de la enfermedad. El principio ético dominante que se aproxima mas a las opiniones de los genetistas Mexicanos es la no maleficiencia, mas que la autonomía individual (27).

El consejero debe ser honesto en sus límites de conocimiento y saber referir al consultante cuando es incapaz de llenar sus necesidades, se debe evitar aprovecharse de él para beneficios, ganancias o interés personales.

\section{Comentarios finales}

Dada la diversidad de mecanismos identificados para la producción de malformaciones y enfermedades genéticas, el AG resulta cada vez mas complejo. Quien proporciona la información debe tenerlos presente para poder proporcionar la orientación adecuada para cada caso. La comunicación con la familia debe hacerse de una manera clara, varía de paciente a paciente y depende de factores que incluyen su nivel de conocimientos general y científico, y sus características socioculturales. Adecuar el lenguaje que se utiliza para la explicación de cada fenómeno, a una forma comprensible para quien lo recibe, es una prioridad en el proceso del AG.

Debe hacerse énfasis en que el proceso del consejo genético debe buscar la facilitación para realizar decisiones autónomas e informadas, por medio de una apreciación correcta de las características hereditarias de la patología, 


\section{Asesoramiento genético.}

integración de la información en un marco de referencia útil y/o mejoramiento emocional del afectado (os) o los miembros de su familia. La percepción individual de riesgo es importante para tomar decisiones acerca de exponerse al mismo, o establecer conductas que lo disminuyan. Debe evitarse conducir al paciente hacia el fatalismo sobre determinado evento adverso y causar ansiedad.

Así mismo, cabe insistir en que se debe aclarar la incertidumbre acerca de la utilidad de las pruebas genéticas a aquellos que consideran riesgos potenciales que alteren su vida o para aquellos que se enfrentan a riesgos incrementados de patologías para las que se dispone de predicción, pero en las que no hay intervención. Sin embargo, cuando una patología genética puede ser tratada, las pruebas genéticas no deben solo ofrecerse, sino ser obligatorias, como en el caso del tamiz neonatal.

Una propuesta surgida de una reunión de expertos en genética, durante el Noveno Congreso Internacional de Genética Humana realizado en Brasil en 1996, para la estructuración de la educación de postgrado, recomienda entrenamiento para consejeros genéticos. Éstos podrían ser varios tipos de profesionales en salud (médicos, enfermeras, psicólogos, biólogos, etc.) entrenados para proporcionar consejo genético bajo la supervisión de genetistas clínicos (20). Es una realidad la necesidad de contar con profesionales entrenados para este fin, dada la diversidad de enfermedades, mecanismos de producción y pruebas diagnósticas que se conocen en la actualidad. Para los médicos familiares activos en la práctica clínica, las necesidades educacionales incluyen la determinación de saber quién necesita AG, como identificar a los pacientes y ayudarlos a entender que esperar del AG. Esto se lograría por medio de conferencias, cursos y publicaciones acerca de este tema (28).

\section{AGRADECIMIENTO.}

Financiado a través del convenio CONACYT SISIERRA 970302.

\section{REFERENCIAS.}

1.- Reed S. A short history of genetic counseling. Soc Biol $1975 ; 22: 332-9$.

2.- Ad Hoc Comitte on Genetic Counseling, American Society of Human Genetics. Genetic Counseling. Am J Hum Genet 1975; 27:240-2.

3.- Guízar-Vázquez JJ. Asesoramiento Genético. En: Guízar- Vázquez J. Genética Clínica. Diagnóstico y manejo de las enfermedades hereditarias. 2nd. ed. México: Manual Moderno; 1994. p. 649-62.

4.- Yoom PW, Olney RS, Kloory MJ, Sappenfield WM, Chavez GF, Taylor D. Contribution of birth defects and genetic diseases to pediatric hospitalizations. Arch Pediatr Adolesc Med 1997; 151:1096-103

5.- Lisker R, Armendares S. Introducción a la genética humana. México: Manual Moderno; 1994. p. 69-105.

6.- Salamanca F. Citogenética humana. Fundamentos y aplicaciones clínicas. México: Editorial Médica Panamericana; 1988. p. 83-92.

7.- Marteau T, Drake H, Bobrow M. Counsellin following diagnosis of a fetal abnormality. J Med Genet 1994; 31 : 864-7.

8.- Marteau T, Drake H, Reid M, Feijoo M, Soares M, Nippert I, Nippert P, Bobrow M. Counsellig following diagnosis of fetal abnormality: a comparison between German, Portuguese and UK geneticist. Eur J Hum Genet 1994; 2:96-102

9.- Carnevale A, Lisker R, Villa AR, Armendares S. Attitudes of Mexican geneticists towards prenatal diagnosis and selective abortion. Am J Med Genet 1998; 75:426-31.

10.- Zavala C, Saavedra D, Samperio-Sánchez CM. Differences in recurrence risk for siblings for cleft lip and /or palate depending on the degree of the malformation and on family history. Rev Inv Clin (Méx) 1983; 35: 4953.

11.- Polifka JE, Friedman JM. Clinical teratology: identifying teratogenic risks in humans. Clin Genet 1999: 56;409-20.

12.- Erickson RP, Lewis SE. The new human genetics. Environ Mol Mut 1995; 25 (suppl 26):7-12.

13.- Ashley CT Jr, Warren ST. Trinucleotide repeat Vol. 12/No. 3/Julio-Septiembre, 2001 


\section{Pinto-Escalante, JM Ceballos-Quintal, I Castillo-Zapata, MT de J López-Avila.}

expansion and human disease. Ann Rev Genetics 1995; 29:703-28.

14.- Frank SA, Hurst LD. Mitochondria and male disease. Nature 1996; 383:224.

15.- Nils-Gran L, Clayton DA. Molecular Genetic aspects of human mitochondrial disorders. Ann Rev Genetics 1995; 29:151-78.

16.- Lalande M. Parental imprinting and human disease. Ann Rev Genet 1997; 30:173-95.

17.- García-Escobar-Morales B. Síndrome de Prader-Willi: Hipotonía, hipogonadismo, obesidad, retraso mental, manos y pies cortos. En: Guízar-Vázquez JJ, Zafra de la Rosa GF, editores. Atlas diagnóstico de síndromes genéticos. México: Manual Moderno; 1999. p. 336-9.

18- Zafra de la Rosa G. Síndrome de Angelman. En: Guízar-Vázquez JJ, Zafra de la Rosa GF, editoeres. Atlas diagnóstico de síndromes genéticos. México: Manual Moderno; 1999. p. 340-1.

19.- Erickson RP, Lewis SE. The new human genetics. Environ Mol Mut 1995; 25 (suppl 26):7-12.

20.- Bowles-Biesecker B, Marteau TM. The future of genetic counselling: A international perspective. Nature genetics 1999; 22:133-7.

21.- Penchaszadeh VB, Beiguelman B. Medical genetic services in Latin America: report of a meeting of experts. Pan Am J Public Health 1998; 3: 409-20.

22.- Annas GJ. Generic consent for genetic screening. New Eng J Med 1994; 330: 1611-3.

23.- Kaback MM. Perspectives in genetic screening. Int $\mathbf{J}$ Assess Health Care 1994; 10:592-603.

24.- Knoppers BM, Chadwick R. The human Genome Project: Under an international ethical microscope. Science 1994; 265:2035-6.

25.- Lisker R, Carnevale A, Armendares S. Mexican geneticists' views of ethical issues in genetics testing and screening. Are eugenic principles involved? Clin Genet 1999; 56:323-7.

26.- WHO Scientific group. Control of hereditary diseases. World Health Organ Tech Rep Ser 1996; 865:1-84.
27.- Lisker R, Carnevale A, Villa JA, Armendares S, Wertz DC. Mexican geneticists' opinions on disclosure issues. Clin Genet 1998; 54:321-9.

28.- Fetter MD, Doukas DJ, Dinh-Phan L. Family physicians' perspectives on genetics and the human genome project. Clin Genet 1999; 56:28-34.

\section{Revista Biomédica}

